\section{Nonparametric regression analysis: A program package for use on a computer terminal}

\author{
EDWARD C. SMITH \\ Medium Transformer Department, General Electric \\ Rome, Georgia 30161 \\ and \\ WAYNE W. DANIEL and BRIAN SCHOTT \\ Department of Quantitative Methods \\ Georgia State University, University Plaza \\ Atlanta, Georgia 30303
}

Although regression analysis is one of the most widely used statistical techniques required by researchers, the assumptions underlying the parametric regression inferential methods are not always met. A number of nonparametric regression procedures are now available, but manual application is generally a tedious undertaking unless the samples are quite small.

The computer program package described here contains programs for the following five nonparametric regression procedures:

(1) Point estimate of slope and y-intercept. The Brown-Mood (Brown \& Mood, 1951; Mood, 1950) method is used to calculate the sample slope and $y$ intercept from raw data. A method due to Theil (1950c) is used to calculate the slope. The program also provides a scatter diagram of the raw data.

(2) Hypothesis tests about the slope and $y$-intercept. This program simultaneously tests the null hypothesis that the population $y$-intercept, $\alpha$, is equal to some $\alpha_{0}$ and that the population slope, $\beta$, is equal to some $\beta_{0}$, using a method proposed by Brown and Mood (Brown \& Mood, 1951; Mood, 1950). The Brown-Mood and Theil (1950a) methods are used to test $\mathrm{H}_{0}: \beta=\beta_{0}$. A scatter diagram is also provided.

(3) A confidence interval for the population slope. This program constructs a two-sided confidence interval for the population slope parameter using the method proposed by Theil $(1950 \mathrm{~b}, 1950 \mathrm{c})$. The user may choose from among the following five confidence coefficients: $.99, .98, .95, .90, .80$.

(4) $A$ test for parallelism. The test statistic proposed by Hollander (1970) for testing the null hypothesis that two regression lines are parallel is computed by this program.

(5) $A$ confidence interval for the difference between two population slopes. This program computes a point estimate and constructs a two-sided confidence interval (any confidence coefficient between .90 and .99 ) for the difference between two slope parameters. The method is due to Hollander and Wolfe (1973).

Each of the programmed procedures is described in the book by Daniel (1978), which also contains tables of the relevant test statistics.

Input. Input to the programs is via computer terminal. Programs 1 through 3 accept up to 50 pairs of observations, and Programs 4 and 5 accept up to 50 pairs of observations for each of two samples (larger samples may be accommodated by changing DIMensions). Each observation must fall within the range -9998.99 to +9998.9. The user supplies sample size, confidence coefficients, the null hypothesis, and similar inputs.

Output. The output for each of the programs is as follows:

(1) (a) Sample slope and y-intercept by the method of Brown and Mood. (b) Sample slope by Theil's method. (c) Scatter diagram.

(2) (a) Test statistic for simultaneous testing of $\mathrm{H}_{0}: \alpha=\alpha_{0}$ and $\mathrm{H}_{0}: \beta=\beta_{0}$ by method of Brown and Mood. (b) Test statistic for testing $\mathrm{H}_{0}: \beta=\beta_{0}$ by method of Brown and Mood. (c) Test statistic for testing $\mathrm{H}_{0}: \beta=\beta_{0}$ by Thiel's method. (d) Scatter diagram.

(3) Upper and lower confidence limits for the population slope parameter for any of the confidence coefficients $(.99, .98, .95, .90$, or .80$)$ by Theil's method.

(4) Test statistic for testing the null hypothesis that two regression lines are parallel by Hollander's method.

(5) Point estimate and upper and lower confidence limits (any confidence coefficient between .90 and .99) for the difference between two slope parameters by the method of Hollander and Wolfe.

Computer and Language. The programs are written in BASIC and may be implemented on any system with a BASIC interpreter with only the "PRINT USING" and "MAT READ" enhancements.

Availability. A program listing and instructions are available free from Professor Wayne W. Daniel, Department of Quantitative Methods, Georgia State University, University Plaza, Atlanta, Georgia 30303.

\section{REFERENCES}

Brown, G. W., \& Mood, A. M. On median tests for linear hypothesis. In J. Neyman (Ed.), Proceedings of the second Berkeley symposium on mathematical statistics and probability. Berkeley and Los Angeles: University of California Press, 1951. Pp. 159-166.

DANIEL, W. W. Applied nonparametric statistics. Boston: Houghton Mifflin, 1978.

Hollander, M. A distribution-free test for parallelism. Journal of the American Statistical Association, 1970, 65, 387-394.

Hollander, M., \& Wolfe, D. A. Nonparametric statistical methods. New York: Wiley, 1973.

Mood, A. M. Introduction to the theory of statistics. New York: McGraw-Hill, 1950. 
THEIL, H. A rank-invariant method of linear and polynomial regression analysis, I. Nederlandse Akademie van Wettenschappen, Proceedings (Series A), 1950, 53, 386-392. (a)

THEILL, H. A rank-invariant method of linear and polynomial regression analysis, II. Nederlandse Akademie van Wettenschappen, Proceedings (Series A), 1950, 53, 521-525. (b)
THEIL, H. A rank-invariant method of linear and polynomial regression analysis, III. Nederlandse Akademie van Wettenschappen, Proceedings (Series A), 1950, 53, 1397-1412. (c)

(Accepted for publication April 18, 1978.) 